\title{
Power Restoration in Distribution Network Using MST Algorithms
}

\author{
T. D. Sudhakar \\ St Joseph's College of Engineering, Chennai, \\ India
}

\section{Introduction}

In this new era electric power has become a fundamental part of the infrastructure of modern society, with most of daily activity is based on the assumption that the desired electric power is readily available for utilization. In the near future, electric supply to houses, offices, schools and factories is taken for granted. The complex power distribution system provides the required electricity to the customers.

The transfer of power from the generating stations to the consumers is known as an electric supply system (figure 1). It consists of three principal components, namely the generating station, transmission lines and distribution networks. The power is generated at favorable places which are quite far away from the customers. The power is produced and transmitted using a 3 phase 3 wire alternating current (A.C.) system and it is distributed using a 3 phase 4 wire A.C. system.

The distribution network components are the distribution substation, the primary feeder, distribution transformers, secondary distribution transformers, sectionalizing switches, tie switches and the services.

The network carries electricity from the transmission systems and delivers it to consumers at the load centres through a number of power lines (branches). Switching on and off of these power lines makes the power to flow in the power distribution network.

\section{Power restoration in distribution system}

The power distribution network can undergo outages, which may be forced or scheduled. Forced outages take place due to any faults in the network, whereas scheduled outages happen because of maintenance work. The various outages that occur in the distribution network are :

- $\quad$ Outage of the primary feeders

- Outage of the distribution transformers

- Outage of the distribution line

During outages, the supply of power is either partially or completely isolated from the feeder to the load centres. This deficit of power supply has to be the minimized. To achieve 


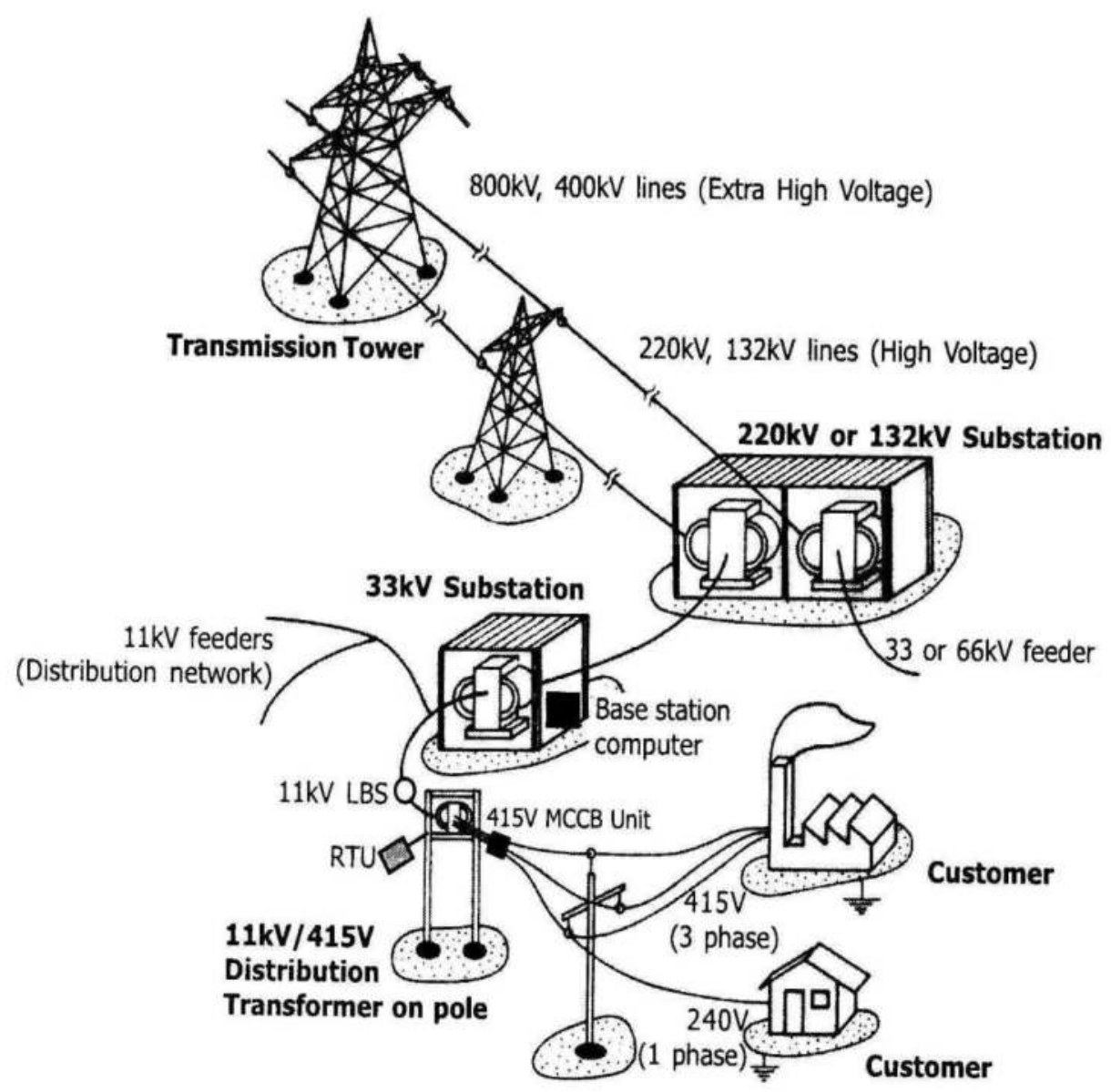

Fig. 1. Basic Electric Supply System

this goal, a proper switching sequence of power lines in the power distribution network is required. Sometimes, the load cannot be served to the customers; in which case, the loads are shed for the least priority customers.

The power distribution network in general, is built as an interconnected mesh network as shown in figure 2. Bus1, bus2 and bus3 indicate the feeder buses and the number in the circle indicates the load buses. The marking Si indicates the distribution branch i, that is used to transfer the power from one bus to another. Feeders in a distribution system have a mixture of types of loads, such as Very Important Person (VIP) \& essential, industrial, commercial and domestic consumer loads. The peak load on feeders occurs at different times of the day, depending upon the type of load, making certain feeders to get heavily loaded and certain others to get lightly loaded. In such a practical situation, the reconfiguration based redistribution of the load amongst the feeders should attempt to evenly distribute the loads in the feeder. 


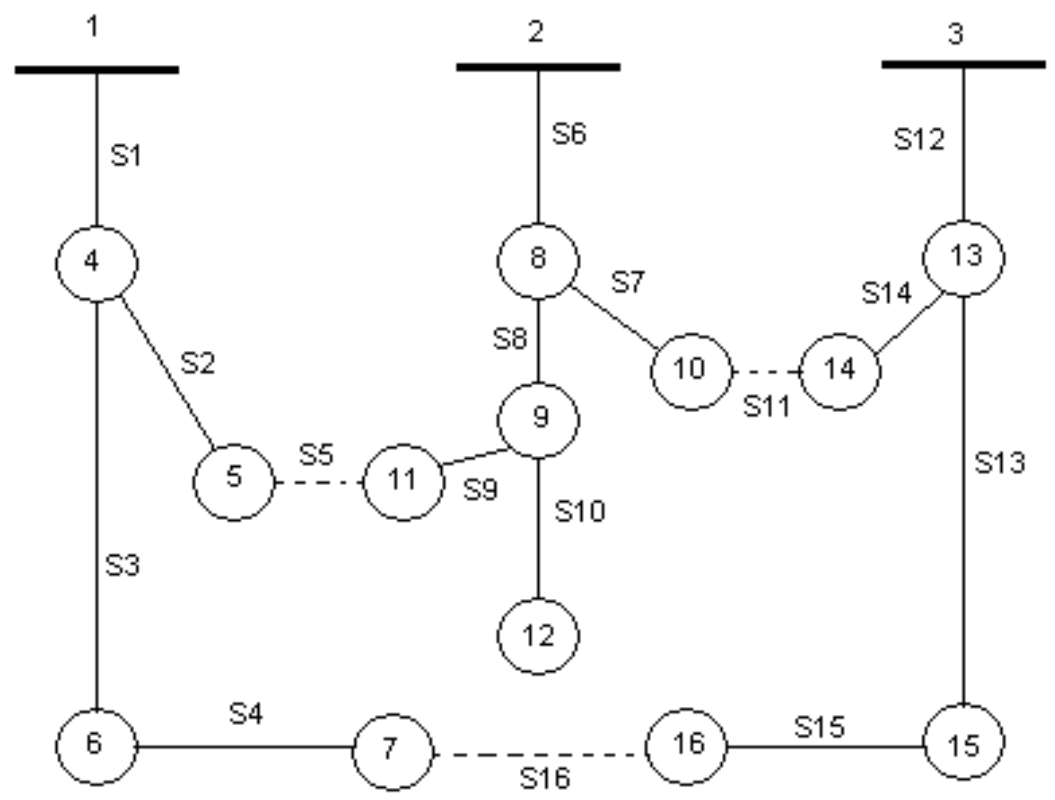

Fig. 2. Basic Power Distribution System

The branches in the power distribution network are normally configured radially for the effective coordination of their protective systems and such radiality is considered here. These networks are divided into a number of subsystems, which contain a number of normally closed switches (sectional switches) and normally open switches (tie line switches). These switches are operated during conditions of maintenance, dispatch and abnormalities. The aim of this switching operation is to reschedule the loads more effectively and improve the reliability of the distribution network. By changing the status of the switches, the topology of the power distribution network is reconfigured, and the resulting line currents and losses are redistributed, with a change in the bus voltage. These parameters of the network are obtained using Backward Sweep Power Flow algorithm.

In the case of an outage in any part of the system, it is imperative to restore the power system to an optimal target of the network configuration. The problem of obtaining a target network by switching is referred to as power system restoration. Power restoration after an outage usually refers to an emergency situation and the resultant plan should meet the following requirements:

- $\quad$ Restore as many loads as possible while considering priority customers

- Not cause violations in either engineering or operating constraints

- Outline a feasible sequence of operations to reach the final configuration

- Power balancing should be done

- $\quad$ Reached in a short time

- Radial network structure. This requirement is based on the feeder design for ease in fault location, isolation and protective device coordination.

- No components must be overloaded 
The implementation of power restoration in a vast distribution system is thus a complicated combinatorial optimization problem because there are a great number of switches in the distribution system. It may take a long time using combinatorial optimization algorithm to reach a feasible restoration plan satisfying all practical constraints. An efficient way of achieving this would be to operate those switches that cause minimum loss and satisfy the voltage, current and other constraints. The major constraints to be satisfied in the distribution network are :

- Load allocation of the Feeder

- Voltage fluctuation

- Customer priority

- Reliability of the network

- Security of the network

- Distributed generation

- Harmonics due to intermittent switching

- Capacitor switching,

- Sudden increase of load and

- Failure of automated communication technology

Therefore, the dispatchers at many utilities tend to use their experience to narrow down and reach a proper restoration plan in a short period. This area has received a lot of attention by the researchers in the past three decades as evidenced by the number of publications (Sudhakar et. al 2011)

With the fast-paced changing technologies in the power industry, novel references addressing new technologies are being published. The automation of restoration of distribution power gained significance in the late eighties (Adibi and Kafka 1991). The state of the art methods used to solve the power system restoration for distribution system problems include Heuristic search (Morelato and Monticelli 1989), Expert system (Hotta et al 1990), and Knowledge based system (Matsumoto et al 1992). Due to the advancement in mathematics, new algorithms were developed to solve the restoration problem in distribution network. It mainly consisted of Artificial Neural Networks (Hoyong Kim et al 1993), Fuzzy Logic control (Han-Ching kuo and Yuan Yih Hsu 1993), Genetic Algorithm (Gregory Levitin et al 1995), Artificial Intelligence (Rahman 1993), Petri net (Fountas et al 1997), Tabu search (Toune 1998), Optimization (Nagata and Sasaki 2002), Ant colony search algorithm (Mohanty et al 2003) and Particle Swarm Optimization (Si-Qing Sheng et al 2009).

The main drawback faced in using the above methods, was the difficulty in identifying all the distribution branches used for the power to flow, after an outage for which predefined rules were used. To overcome this drawback, hybrid models such as fuzzy GA model (YingTung Hsiao and Ching-Yang Chien 2000), was tried. To solve a complex combinatorial problem, the time required for solving the restoration problem using any of the above said methods is high. Now if hybrid models are used then the time required to obtain a solution is still higher. As a result it has become mandatory to identify the radial path of power flow with least mathematical efforts.

Most of the work reported focused on constraints like voltage limits, radiality and feeder capacity as the time required to obtain a restoration plan is more. To maintain these 
constraints, load shedding is done immediately. Load shedding option would imply loss of supply to essential loads such as medical facilities. If the time consumed is less; then the line losses and feeder capacity based on internal load division priority can be considered. To obtain the solution of the restoration problem without any iterative procedure, a graph theory based minimum spanning tree (MST) methodology proposed.

\section{Graph theory}

Graphs, the basic subject studied by the graph theory are abstract representations of a set of objects, where some pairs of objects are connected by links. The interconnected objects are represented mathematically as vertices, and the links that connect some pairs of vertices are called edges. Typically, a graph is depicted in a diagrammatic form as a set of dots for the vertices, joined by lines or curves called the edges. The vertices are also called nodes or points, and the edges are called lines.

A graph can be classified into two types namely an undirected and directed graph. A graph may be undirected, meaning that there is no distinction between the two vertices associated with each line, or its lines; or directed, meaning there is a distinction between one node and another. Table 1 shows the terminology for proceeding through the graph theory.

\begin{tabular}{|l|l|}
\hline Term & Meaning \\
\hline a & Vertex or node \\
\hline a & $\begin{array}{l}\text { The line joining two nodes or vertices is called an line. Since the line } \\
\text { doesn't show the direction it is an undirected graph. }\end{array}$ \\
\hline a & $\begin{array}{l}\text { An line having a weight } 5 \text { being connected between the node } 1 \text { and } \\
\text { node } 2\end{array}$ \\
\hline
\end{tabular}

Table 1. Symbols used in minimum spanning tree

Fig. 3. shows an example network with 6 nodes and 10 lines, which have their respective weights.

\subsection{Minimum spanning tree}

In the mathematical field of the graph theory, a spanning tree $\mathrm{T}$ of a connected, undirected graph $\mathrm{G}$ is a tree composed of all the vertices and some (or perhaps all) of the lines of G. That is, every node lies in the tree, but no cycles (or loops) are formed. A spanning tree of a connected graph $\mathrm{G}$ can also be defined as a maximal set of lines of $\mathrm{G}$ that contains no cycle, or as a minimal set of lines that connect all the vertices. For a connected graph with $\mathrm{V}$ nodes, any spanning tree will have the $\mathrm{V}-1$ lines.

Given a connected, undirected graph, a spanning tree of that graph is a subgraph, which is a tree and connects all the vertices together. A weight is assigned to each line, whose value represents how unfavorable it is for the considered task. Individual weights of lines in a spanning tree decide its weight. The total sum of all the weights of the lines in a particular spanning tree is its weight. 


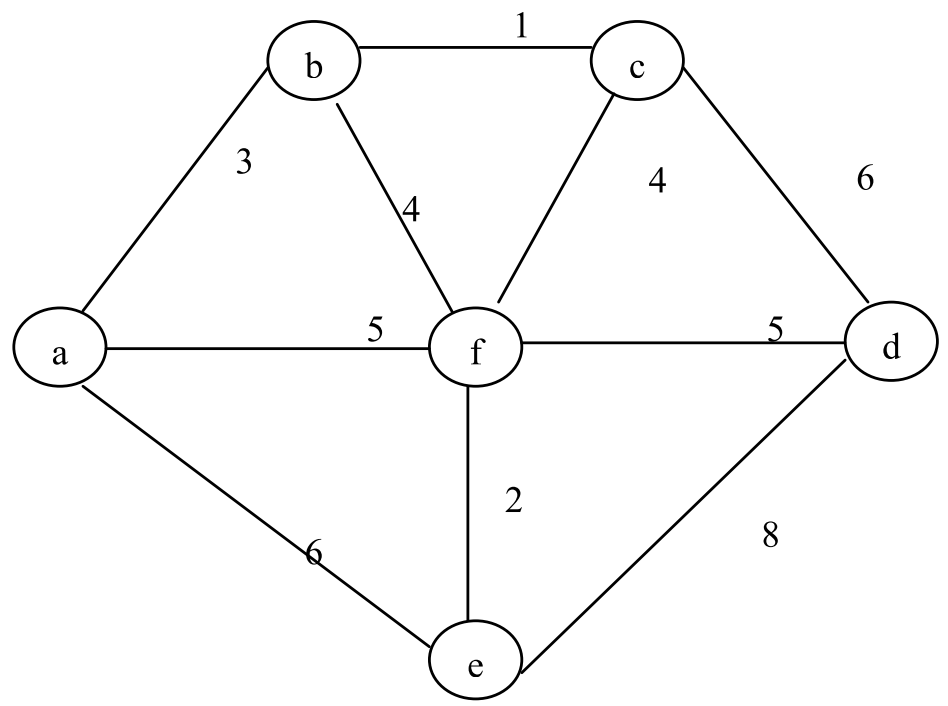

Fig. 3. Example network for MST problem

A minimum spanning tree (MST) or minimum weight spanning tree is then a spanning tree with a weight less than or equal to the weight of every other spanning tree. This concept is often used in routing. The algorithms to find MST, a graph search algorithm that solves the single-source shortest path problem for a graph with non-negative line path weights, produces a shortest path tree. For a given source node in the graph, the algorithm finds the path with the lowest weight (i.e., the shortest path) between that node and every other node. For example, if the nodes of the graph represent cities, and the line path weights represent the driving distances between pairs of cities connected by a direct road then MST can be used to find the shortest route between one city and all other cities. The main advantage of using the MST is that the optimum solution is obtained in a single stage.

\subsection{Algorithms for finding MST}

This section presents the proposed MST algorithm. Each algorithm is explained with a common example. A single graph can have many different spanning trees. If an exhaustive search approach to construct an MST is tried, two serious drawbacks arise. First, the number of spanning trees grows exponentially with the graph size; second, generating all the spanning trees for a given graph is not easy. To overcome these drawbacks the MST algorithms are proposed. The MST algorithms are further classified as line based MST algorithms and node based MST algorithms. They are

1. Line based MST algorithms

i. Kruskal's algorithm

ii. Reverse Delete algorithm

2. Node based MST algorithms

i. Prim's algorithm

ii. Dijkstra's algorithm 


\subsection{Kruskal's algorithm}

Kruskal's algorithm is an algorithm in graph theory presented in 1956 (Anany Levitin (2009)) that finds a minimum spanning tree for a connected weighted graph. This means that it finds a subgraph of the lines that form a tree. It includes every node of the network and the total weight of all the lines in the tree is minimized. To implement the Kruskal's algorithm, two conditions have to be satisfied. First the weight of the lines in a graph is arranged in the increasing order. Second an empty subgraph $\mathrm{T}$ (called Traversal matrix) is created.

Then, the algorithm considers a line, based on the order of increasing weight. If a line $(u, v)$ $(u, v$ are the starting \& ending node of a line) does not form a cycle along with the existing lines in the subgraph $(\mathrm{T})$ then the line $(u, v)$ is added to the subgraph $(\mathrm{T})$. Then the line $(u, v)$ is discarded. If a line is added to the subgraph then the counter is incremented. It is checked that the counter value is equal to $\mathrm{V}-1$, where $\mathrm{V}$ is the number of nodes. If it is true, the procedure is stopped; otherwise, the process continues.

The Kruskal's algorithm is applied to the example network as shown in figure 3. The step by step procedure of the algorithm is discussed by Sudhakar et al (2011). The resultant traversal matrix with a weight of 15 is

$$
\begin{array}{r}
T=[\mathrm{b} \mathrm{c} \\
\mathrm{f} \mathrm{e} \\
\mathrm{b} \mathrm{a} \\
\mathrm{bf} \\
\mathrm{f} \mathrm{d}]
\end{array}
$$

At the termination of the algorithm, the traversal matrix forms a MST of the graph. Based on this process, a pseudo code of the algorithm is developed and is as follows,

Pseudocode for the Kruskal's algorithm:

Sort $\mathrm{E}$ in increasing order of edge weights and the sorted edges are in A.

- Initialize the set of tree edges and its size, T.

- Counter $\leftarrow 0$

- While counter $<|\mathrm{V}|-1$

- If $\mathrm{T} U(u, v)$ is acyclic

- $\mathrm{T} \leftarrow \mathrm{T} \mathrm{U}(u, v)$

- Counter $\leftarrow$ Counter+1

Return T

\subsection{Reverse Delete algorithm}

The Reverse-Delete algorithm is an algorithm in graph theory used to obtain a minimum spanning tree from a given connected, line-weighed graph. The Reverse-Delete algorithm starts with the original graph and deletes lines from it. The Reverse-Delete algorithm ensures connectivity in the graph before deletion. Since the algorithm only deletes lines 
when it does not disconnect the graph, any line removed by the algorithm forms a cycle prior to the deletion. Since the algorithm starts from the maximum weighted line and continues in descending order, the line removed from any cycle is the maximum weighted line in that cycle. Therefore, according to the definition of a minimum spanning tree, the lines removed by the algorithm are not in any minimum spanning tree.

The Reverse-Delete algorithm is applied to the example network as shown in figure 3. The step by step procedure of the algorithm is discussed by Sudhakar et al (2011). Thus, the minimum weight for traversing the graph is 15 and the resultant network is shown in figure 4 .

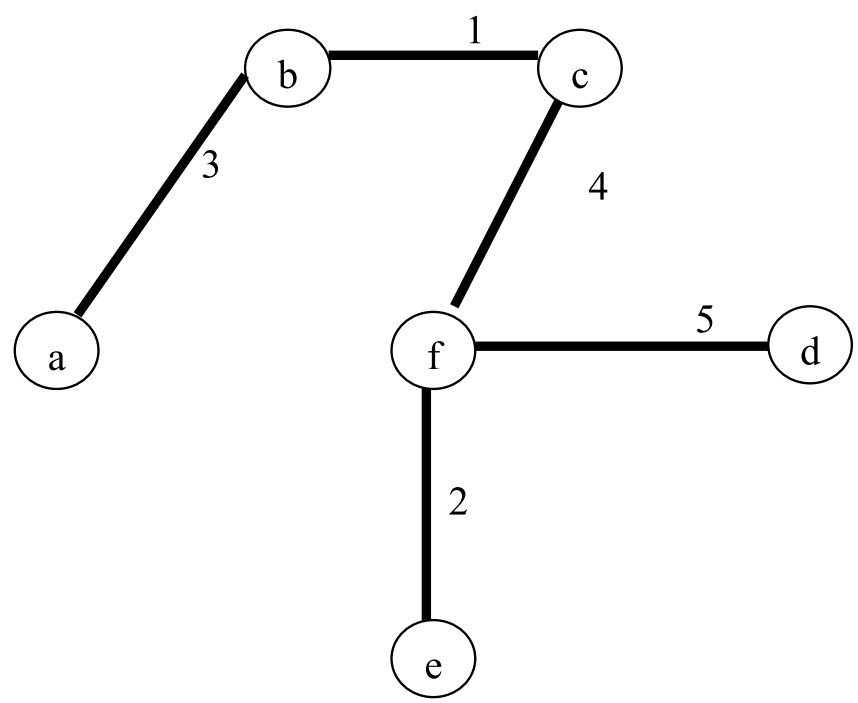

Fig. 4. Final Result of Reverse-Delete algorithm

Based on this procedure a pseudo code of the algorithm is formed as follows :

Pseudocode for the Reverse-Delete algorithm:

Function Reverse Delete (edges [T])

Sort $\mathrm{T}$ in decreasing order

Define an index $\mathrm{i} \leftarrow 0$

while $\mathrm{i}<\operatorname{size}(\mathrm{T})$

Define edge temp $\leftarrow \mathrm{T}[\mathrm{i}]$

delete $\mathrm{T}[\mathrm{i}]$

if temp.v1 and temp.v2 are not connected to the tree

$$
\begin{aligned}
& \mathrm{i} \leftarrow \mathrm{i}] \leftarrow \text { temp } \\
& \leftarrow+1
\end{aligned}
$$

return edges[T]

\subsection{Prim's algorithm}

The algorithm was developed in 1930 by the Czech mathematician, Vojtech Jarnik, and later independently, by the computer scientist, Robert C. Prim, in 1957 (Anany Levitin (2009)). 
This algorithm finds a subset of the nodes that form a tree that includes every node, where the total weight of all the lines in the tree is minimized.

The Prim's algorithm constructs an MST through a sequence of expanding subtrees. The initial subtree in such a sequence consists of a single node selected arbitrarily from the set $V-V_{T}$ of the graph's nodes. In the following steps, the current tree expands, by simply getting attached to a nearer node with less weight. The algorithm stops, after all the graph's nodes have been included in the tree being constructed. Since the algorithm expands a tree by exactly one node on each of its steps, the total number of such steps is $V-1$, where $V$ is the number of nodes in the graph.

The nature of the Prim's algorithm makes it necessary to provide two data values for every other unselected node. The data values are provided through two arguments : first will be the unselected node's $\left(V-V_{T}\right)$ connectivity to the currently selected node $\left(V_{T}\right)$. It will be nil '' if no connectivity exists. The second entry (distance label) will be the respective weight. If there is no connection then the value will be $\infty$. With such labels, the smallest distance label in the set $V-V_{T}$, is selected and added in the selected nodes list.

After a node $e^{*}$ is identified which is to be added to the tree, the following operations have to be performed:

- $\quad$ Move $e^{*}$ from the set $V-V_{T}$ to the set of selected nodes $V_{T}$.

- $\quad$ Based on the nodes in set $V_{T}$, the weights of the node in $V-V_{T}$ are updated.

- For each remaining node $u$ in $V-V_{T}, e^{*}$ is selected which has the minimum weight

- The $e^{*}$ is the next node to be added to the current tree T and the node $e^{*}$ is added in $V_{T}$

The Prim's algorithm is applied to the example network as shown in figure 3 . The step by step procedure of the algorithm is discussed by Sudhakar et al (2011). Thus, the minimum weight for traversing the graph is 15 and the resultant network is shown in figure 5.

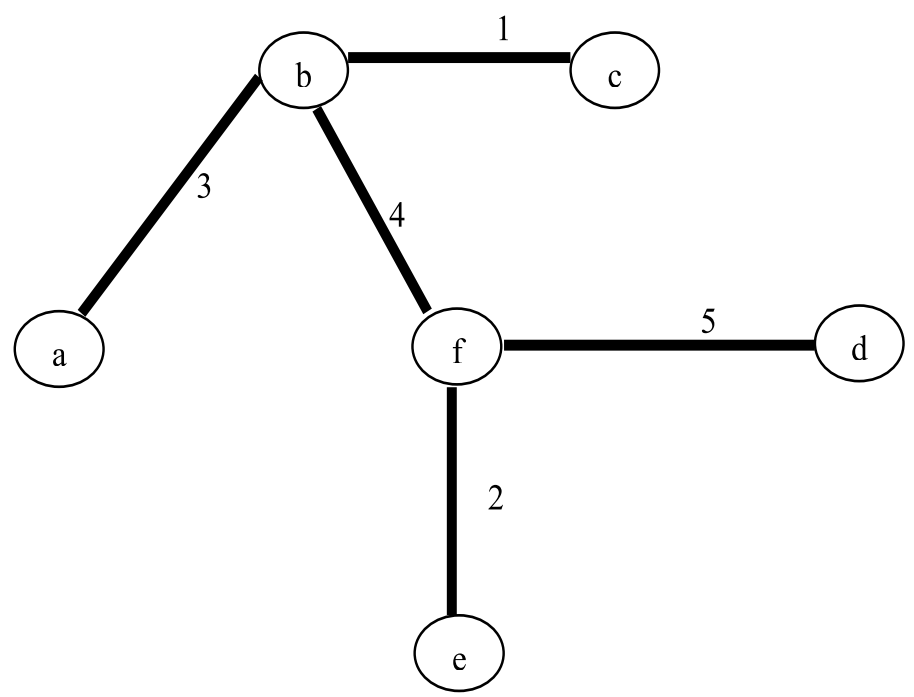

Fig. 5. Result of Prim's algorithm 
Based on this procedure a pseudo code of the algorithm is formed as follows :

\section{ALGORITHM Prim(G)}

/ / Prim's algorithm for constructing an MST

//Input: A weighted connected graph $\mathrm{G}=(V, E) / / \mathrm{V}$ is node; $\mathrm{E}$ is line

//Output: $\mathrm{E}_{\mathrm{T}}$, the set of lines composing the MST of $G$

$/ /$ the set of tree nodes can be initialized with any node

$E_{T} \leftarrow \emptyset$

for $i=1$ to $(V-1)$ do

find a minimum-weight line $e^{*}=\left(v^{*}, u^{*}\right)$ among all the lines $(v, u)$

such that $v$ is in $V_{T}$ and $u$ is in $V-V_{T}$

return $E_{T}$

$$
\begin{aligned}
& V_{T} \leftarrow V_{T} \cup\{u\} \\
& E_{T} \leftarrow E_{T} \cup\{v\}
\end{aligned}
$$

\subsection{Dijkstra's algorithm}

The Dijkstra's algorithm, was conceived by the Dutch computer scientist Edsger Dijkstra in 1959 (Anany Levitin (2009)). This algorithm uses traversal matrix [T]. It indicates the distance from a single node to all the other nodes including it. In the considered example of figure 3, when node ' $a$ ' is considered first, its distance from it and all nodes from a through $\mathrm{f}$ will form the initial entries of [T]. The same procedure is repeated till all the nodes are selected and the final network with a total weight of 20 as shown in figure 6. algorithm is applied to the example network as shown in figure 3. The step by step procedure of the algorithm is discussed by Sudhakar et al (2010). Thus, the minimum weight for traversing the graph is 20 and the resultant network is shown in figure 6.

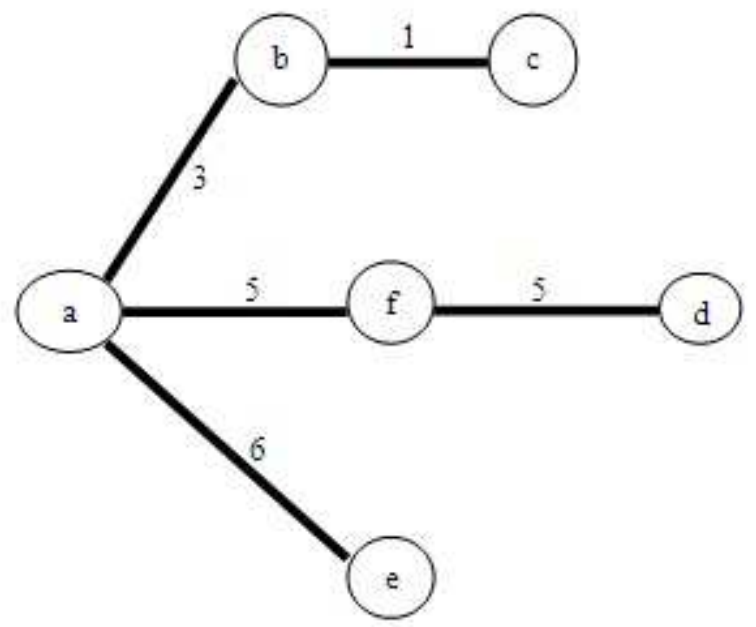

Fig. 6. Resultant network of Dijkstra's algorithm 
Based on this modus operandi, a pseudo code of the algorithm is developed as follows,

Pseudocode for the Dijkstra's algorithm:

function Dijkstra(Graph, source):

// Initializations

for each node $v$ in Graph:

// Unknown distance function from s to $v$

$\operatorname{dist}[v]:=$ infinity previous $[v]:=$ undefined

$/ /$ Distance from $s$ to $s$

dist [source] : $=0$

$/ /$ Set of all unvisited vertices

$Q:=\operatorname{copy}($ Graph $)$

// The main loop

while $Q$ is not empty:

// Remove best node from the priority queue;

$/ /$ returns to the source after first step

$u:=$ extract_min $(Q)$

for each neighbor $v$ of $u$ :

alt $=\operatorname{dist}[u]+$ length $(u, v)$

$/ / \operatorname{Relax}(u, v)$

if alt $<\operatorname{dist}[v]$

$\operatorname{dist}[v]:=$ alt

previous $[v]:=u$

\subsection{Comparison of the results}

The four algorithms are applied to the example network of figure 3, and the results are tabulated in table 2 .

\begin{tabular}{|l|l|}
\hline Algorithm & Total weight \\
\hline Prim's algorithm & 15 \\
\hline kruskal's algorithm & 15 \\
\hline Dijkstra's algorithm & 20 \\
\hline Reverse Delete algorithm & 15 \\
\hline
\end{tabular}

Table 2. Comparison of MST algorithms for example network

In the case of the Dijkstra's algorithm, the total weight of the resultant network is more than that of the other three algorithms; but for the case of node ' $\mathrm{f}$ ' from the starting node ' $\mathrm{a}$ ' the total weight using the Dijkstra's algorithm is 5 which is smaller than the value 7 obtained using the other three algorithms. As a result we cannot neglect the Dijkstra's algorithm as an inefficient method.

\subsection{MST applications}

Graph theory based on MST approach has been discussed in various papers for various applications. Lin Ming Jin \& Shu Park Chan (1989) presents an algorithm for finding the shortest path for power routing (DC) between two nodes in an electrical network used in the 
airlines. Hiroyuki Mork, Senji Tsuzuki (1991) presents mathematically MST for network topological observability analysis. Shun Lin Su et al (1994) dealt with application of the MST for finding the connectivity in VLSI circuits. Cavellucci \& Lyra (1997), presented the minimization of energy losses in distribution systems by applying a general search method to a Brazilian power network. Here outages were not considered as an important factor. Michel Barbehenn (1998) discusses about the application of Dijkstra's algorithm for various applications like airline electrical networks. Ali Shatnawi et al (1999) indicates the use of Floyd - Warshall's based MST to find the time scheduling in the data flow graph of a DSP. Partricia Amancio Vargas (2002) uses the learning classifier system for loss minimization in a power system. Kaigui Xie (2003) calculates the reliability index of radial network using forward search method of MST. TianTian CAi \& Qian Ai (2005) discusses the depth first search method used to find the MST for the optimal placement of the PMU devices in the power system. Distribution reconfiguration algorithm, named Core Schema Genetic Shortest-path Algorithm (CSGSA) proposed by Yixin Yu \& Jianzhong Wu (2002) is based on the weights calculation method for each load condition based on line losses.

The above survey highlighted the extension of the application of graph theory for MV power distribution AC system, which has been attempted at this juncture. Here, the mathematical formulation of Yixin $\mathrm{Yu}$ has been applied to a PDN wherein distribution branch outages have been fully addressed. Thus to obtain the restoration plan quickly without any iterative procedure, a graph theory based methodology using MST algorithms is proposed here. Four algorithms based on graph theory are used to restructure the PDN by considering the distribution branch outage which forms the major contribution of this work.

The MST algorithm identifies all the possible paths for the power to flow and obtains only one solution. In a single iteration the MST algorithm overcome the radiality constraint. Since, MST algorithm gives a path of minimum impedance, the line losses will be minimum with the result no separate loss minimization procedure is required here. The solution of MST algorithm minimizes the solution time and as a result loss minimization and load shedding with internal priorities are included in the proposed work. Thus, in a minimum time a power system restoration solution is obtained, which will not lead to cascaded outage.

\section{Restoration problem}

An outage degrades the most important function of an electrical system, that of supplying the customers, and thus has a radical influence on the operating objectives. Whenever power supply interruption occurs in distribution systems due to an outage, it is imperative to bring back the system promptly to its initial state or to an optimal target network, by switching operations. The problem of obtaining a target network is called as power system restoration, has two prime objectives (a) the number of customers with a restored supply should be the largest possible and (b) the restoration should be accomplished as quickly as possible.

In this section, the network reconfiguration problem for service restoration is discussed in detail. The system is represented on a per phase basis and the load along a feeder section is represented as constant $\mathrm{P}, \mathrm{Q}$ loads placed at the end of the lines. It is assumed that every switch is associated with a line in the system. The network reconfiguration problem for loss 
reduction involves the load transfer between the feeders or substations by changing the position of the switches. The radial configuration corresponds to a 'spanning tree' of a graph representing the network topology.

Given a graph, find a spanning tree such that the problem formulation of the restoration problem is given here

Objective Function is to

\section{Find the Optimal Power Path}

With the following constraints:

i. Maximize the power restored to the isolated area,

$$
\max f=\sum_{K \in B} L_{K} X_{K}
$$

Where $\mathrm{L}_{\mathrm{K}}$ is the load at bus $\mathrm{K}$

$B$ is the total number of buses

$\mathrm{X}_{\mathrm{K}}$ is the decision variable

The position of the sectional and tie line switches is considered here as the binary decision variable. This variable decides whether the switch is open or closed. The binary decision variable, $\mathrm{X}_{\mathrm{K}}$ is defined as

$$
\begin{aligned}
X_{K} & =1 \text { (if the } K^{\text {th }} \text { switch is closed) } \\
& =0 \text { (if the } K^{\text {th }} \text { switch is open) }
\end{aligned}
$$

If there are $\mathrm{m}$ switches in the initial network, there will be $2^{\mathrm{m}}$ on / off switching options. That is, for a two switch network, $2^{2}$ switching options are possible $[(0,0),(0,1)$, $(1,0),(1,1)]$. The resultant decision vector is given by

$$
X=\left[X_{1}, X_{2}, X_{3} \ldots \ldots \ldots \ldots \ldots . . . . . . . . .\right.
$$

For the two switch network the decision vector can be represented as $X=(0,0), X=(0,1)$, $X=(1,0)$ and $X=(1,1)$.

ii. Voltage limits : For each bus bar, the voltage constraints have upper and lower limits. A general expression for voltage constraints would be

$$
\left|V_{\min j}\right| \leq\left|V_{j}\right| \leq\left|V_{\operatorname{maxj}}\right|
$$

where $\left|V_{j}\right|$ is the voltage at $j$ node, $\left|V_{\min j}\right|$ is the minimum permitted voltage at node $j$, $\left|V_{\max }\right|$ is the maximum permitted voltage at node $\mathrm{j}$, and $\mathrm{j}$ belongs to a set of buses where voltage constraints are observed.

iii. Radiality : It is a condition in power distribution network that only one feeder bus feeds a load bus.

iv. Loading constraints : A general expression for loading constraints would be

$$
\mathrm{L}_{\mathrm{i}}<\mathrm{L}_{\mathrm{MP}} \mathrm{i}
$$


where $L_{i}$ is the loading of the network element $i, L_{M P} i$ is the maximum permitted loading of the network element $i$, and $i$ belongs to a set of protected network elements. These protected network elements, are normally lines and feeders that can be protected by actual protection devices, or can be algorithmically protected. However, the fact is that, the considered feeder is not capable of supplying the whole load and hence, cannot be used further for problem solving.

v. Line losses: The total power losses of the network should be minimum.

vi. Feeder capacity: The total capacity of the feeder should not be violated. and

vii. Priority of customers: As the priority of each service zone is determined in advance, which customers would be restored can be determined according to the $\mathrm{L}_{\mathrm{MP}} \mathrm{i}$.

This is a combinatorial optimization problem, since the solution involves the consideration of all possible spanning trees.

\section{Application of the proposed MST algorithms for restoration problem}

MST algorithm is a graph search algorithm that solves the single-source shortest path problem for a graph with non-negative weights, producing a shortest path tree. For a given source vertex (node) in the graph, the algorithm finds the path with lowest impedance (i.e. the shortest path) between the source node and every other node.

In order to achieve a maximum amount of power restored in a radial distribution system, the aim is to identify the appropriate switching options, which consists of all the buses in the network. In the proposed method, the distribution system is considered with all its laterals simultaneously, instead of determining the switching options on loop by loop basis. In applying the graph theory the buses and the feeders are considered as the node, the distribution line is considered as line and the impedance of the distribution line is considered as weight of the line. With this consideration the proposed graph theory based algorithm for the distribution system is :

Step 1. Initial power flow path is stored

Step 2. Get the input data about the amount of loads at each bus, the feeder capacity and the current status of all the lines

Step 3. In case of any outage remove those data from the input data file

Step 4. Check for multi feeder or single feeder

Step 5. If it is single feeder go for step 7

Step 6. If it is multi feeder, then enter the number of feeders

Step 7. Get the MST for the feeders

Step 8. Perform the load flow for the resultant network

Step 9. Check for multi feeder or single feeder

Step 10. If it is single feeder go to step 13

Step 11. Check for feeder overloading condition. If the feeders are overloaded, then load transferring can be done. If load transferring is not possible then perform load shedding. Otherwise if the feeders are not overloaded proceed to step 13.

Step 12. Perform the load flow for the modified conditions

Step 13. Check for over voltage condition. If the voltage limits are violated then perform load shedding otherwise proceed to step 15.

Step 14. Perform the load flow for the modified conditions 
Step 15. Check for over current condition. If the current capacity are violated then perform load shedding otherwise proceed to step 17.

Step 16. Perform the load flow for the modified conditions

Step 17. If all the constraints are satisfied then display the optimal switching sequence

By applying this methodology objective function is solved and the constraints are satisfied. The result of step 7 finds a path for the power to flow after an outage, which satisfies the objective function. This step also satisfies the constraints viz., maximize the power restored to the isolated area, radiality and line losses. The power restored to the isolated area is maximum as the MST obtained after step 7 has all the possible buses available in the network. So all the loads connected to these buses will receive the power. The MST does not allows any closed loop in the network, as a result the MST obtained after step 7 will not have any closed loops, so the radiality constraint is satisfied. The MST network will have a minimum impedance value because the MST is obtained by considering the impedance value of each distribution line. Then the losses of the network will be minimum.

The feeder capacity constraint is mainly applicable for the multi feeder networks. In the case of the single feeder network, the loads are rearranged in the same network whereas in the multi feeder network there are some conditions that the loads of a feeder are transferred to the next feeder nearby. This condition is checked in the step 10. If there is any feeder overloading then load transferring is done. If load transferring is also not possible then load shedding is done based on the priority of the customer's constraint.

Step 13 checks the voltage limit condition and step 15 checks the loading constraint of the network. The maximum allowable limits are based on the network considered. Thus the proposed methodology satisfies all the above said objective function and the constraint.

The proposed methodology is not an iterative procedure, it calculates the amount of the load to be shed at each bus or load transferring between feeder (in the case of multi feeders) three times to satisfy the constraints. The load shed or load transferring has to be performed three times because the MST obtained by step 7 is based on the impedance minimization; it means the load has no influence on the solution. To bring in the effect of the load conditions only the load shedding or load transferring is done. Each time the amount of load to be shed is calculated at each bus and finally all the loads are shed simultaneously.

\section{Results of the proposed MST algorithms for restoration problem}

The original configuration of 33-bus test distribution network shown in figure 7 has a total load capacity of 3.525 MW and 2.3 Mvar. The network consists of 33 buses and 37 branches, where branches S1-S32 and S33-S37 indicate the sectional and tie lines switch respectively. The total impedance of the network for the original configuration having sectional lines is $21.8744+\mathrm{j} 18.1456$ and the loss of the network $0.1869 \mathrm{MW}$ and $0.1240 \mathrm{Mvar}$.

For the given network, the proposed methodology using the four MST algorithms is applied and the switch that should be kept open under normal conditions is tabulated in Table 3. The table indicates the switches those act as tie switches, their total impedance of the resultant network, loss of the network after applying the Kruskal's algorithm, Reverse delete algorithm, Prim's algorithm \& Dijkstra's algorithm and minimum p.u. voltage of the network. 


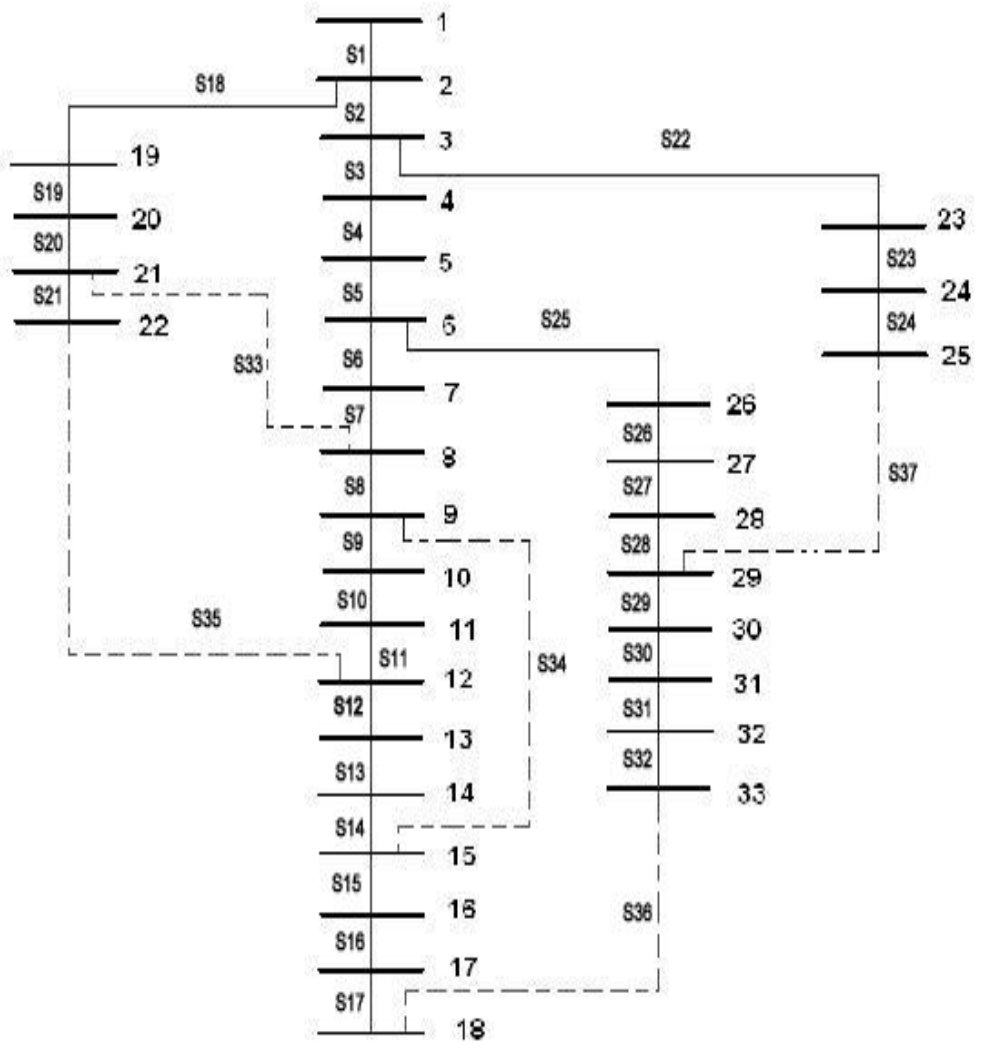

Fig. 7. Thirty three bus single feeder distribution network

\begin{tabular}{|c|c|c|c|c|c|c|c|c|c|c|}
\hline \multirow{2}{*}{ Algorithm } & \multirow{2}{*}{\multicolumn{5}{|c|}{ Tie Switches }} & \multicolumn{2}{|c|}{$\begin{array}{c}\text { Total } \\
\text { Impedance }\end{array}$} & \multirow{2}{*}{$\begin{array}{c}\text { Real } \\
\text { power } \\
\text { loss }\end{array}$} & \multirow{3}{*}{$\begin{array}{c}\begin{array}{c}\text { Reactive } \\
\text { power } \\
\text { loss }\end{array} \\
\text { Mvar } \\
0.1230\end{array}$} & \multirow{3}{*}{ 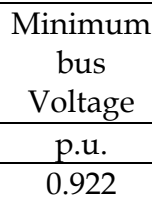 } \\
\hline & & & & & & $\mathrm{R}$ & $X$ & & & \\
\hline KRUSKAL'S & S16 & s27 & S33 & S34 & S35 & 20.52 & 16.49 & 0.1780 & & \\
\hline $\begin{array}{l}\text { REVERSE } \\
\text { DELETE }\end{array}$ & S16 & S27 & S33 & S34 & S35 & 20.52 & 16.49 & 0.1780 & 0.1230 & 0.922 \\
\hline PRIM'S & S16 & S27 & S33 & S34 & S35 & 20.52 & 16.49 & 0.1780 & 0.1230 & 0.922 \\
\hline DIJKSTRA'S & S9 & S14 & S16 & S28 & S33 & 26.15 & 22.46 & 0.1671 & 0.1192 & 0.928 \\
\hline
\end{tabular}

Table 3. Switches that are open and the impedance of the network

From the table it is observed that the resultant losses of the network obtained using the MST algorithms for the normal condition are less when compared to that of the original configuration. In the case of Dijkstra's algorithm it is noted that the impedance value is higher than the total impedance obtained using other three MST algorithm and the initial configuration. The value of the loss is less when compared to other minimum spanning tree algorithms and the initial network. 
For the same network hybrid GA (Jizhong Zhu and Chang (1998)) and heuristic search method (Shirmohammadi and Hong (1989)) are applied and their results are tabulated in Table 4.

\begin{tabular}{|c|c|c|c|c|c|c|c|c|c|c|}
\hline \multirow[t]{2}{*}{ Algorithm } & \multicolumn{5}{|c|}{ Tie Switches } & \multicolumn{2}{|c|}{$\begin{array}{c}\text { Total } \\
\text { Impedance }(\Omega)\end{array}$} & \multirow{3}{*}{$\begin{array}{c}\begin{array}{c}\text { Real } \\
\text { power } \\
\text { loss }\end{array} \\
\text { MW } \\
0.2007\end{array}$} & \multirow{3}{*}{$\begin{array}{c}\begin{array}{c}\text { Reactive } \\
\text { power } \\
\text { loss }\end{array} \\
\text { Mvar } \\
0.1776\end{array}$} & \multirow{3}{*}{$\begin{array}{c}\begin{array}{c}\text { Minimum } \\
\text { bus } \\
\text { Voltage }\end{array} \\
\text { p.u. } \\
0.883\end{array}$} \\
\hline & & & & & & $\mathrm{R}$ & $X$ & & & \\
\hline REFINED GA & S7 & $\mathrm{S} 10$ & S14 & S36 & S37 & 25.38 & 22.31 & & & \\
\hline $\begin{array}{l}\text { HEURISTIC } \\
\text { METHOD }\end{array}$ & S7 & S9 & S14 & S32 & S37 & 24.39 & 21.61 & 0.1984 & 0.1760 & 0.887 \\
\hline
\end{tabular}

Table 4. Switches that are open and the impedance of the network

Using the proposed methodology for a single line outage in 33 - bus network, the results are obtained and tabulated in Table 5.

\begin{tabular}{|c|c|c|c|c|c|}
\hline OUTAGE IN LINE & \multicolumn{5}{|c|}{ SWITCHES THAT ARE OPEN } \\
\hline S1 & & & anno & & \\
\hline S2 & S2 & S8 & S24 & S32 & S34 \\
\hline S3 & S3 & S7 & S9 & S14 & S16 \\
\hline S4 & S4 & S7 & S9 & S14 & S16 \\
\hline S5 & S5 & S7 & S9 & S14 & S16 \\
\hline S6 & S6 & S9 & S13 & S16 & S28 \\
\hline S7 & S7 & S9 & S16 & S28 & S34 \\
\hline S8 & S8 & S15 & S28 & S33 & S34 \\
\hline S9 & S9 & S14 & S16 & S28 & S33 \\
\hline S10 & S10 & S14 & S16 & S28 & S33 \\
\hline S11 & S11 & S14 & S16 & S28 & S33 \\
\hline $\mathrm{S} 12$ & S12 & S9 & S16 & S28 & S33 \\
\hline S13 & S13 & S9 & S16 & S28 & S33 \\
\hline S14 & S14 & S9 & S16 & S28 & S33 \\
\hline S15 & S15 & S9 & S14 & S28 & S33 \\
\hline S16 & S16 & S9 & S14 & S28 & S33 \\
\hline S17 & S17 & S9 & S14 & S28 & S33 \\
\hline S18 & S18 & S13 & S16 & $\mathrm{S} 28$ & S35 \\
\hline S19 & S19 & S13 & S16 & S28 & S35 \\
\hline S20 & S20 & S13 & S16 & S28 & S35 \\
\hline S21 & S21 & S14 & S16 & S28 & S33 \\
\hline S22 & S22 & S9 & S14 & $\mathrm{S} 16$ & S33 \\
\hline S23 & S23 & S9 & S14 & S16 & S33 \\
\hline S24 & S24 & S9 & S14 & $\mathrm{S} 16$ & S33 \\
\hline S25 & S25 & S9 & S14 & S16 & S33 \\
\hline S26 & S26 & S9 & S14 & $\mathrm{S} 16$ & S33 \\
\hline S27 & S27 & S9 & S14 & S16 & S33 \\
\hline S28 & S28 & S9 & S14 & S16 & S33 \\
\hline S29 & S29 & S9 & S14 & S28 & S33 \\
\hline S30 & $\mathrm{S} 30$ & S9 & S14 & S28 & S33 \\
\hline S31 & S31 & S9 & S14 & S28 & S33 \\
\hline S32 & S32 & S9 & S14 & S28 & S33 \\
\hline
\end{tabular}

Table 5. Result for single line outage in 33 bus network 


\subsection{Hardware implementation of the proposed MST algorithms for restoration problem}

For the real time application in the automated world the developed program has to be used with hardware. So the developed methodology is implemented using Verilog HDL. Verilog is a hardware description language (HDL). A HDL is a language used to describe a digital system, for example, a network switch, or any memory or a single flip flop. This means that by using a HDL, one can describe any hardware at any level.

The proposed methodology indicates the ON and OFF status of the switch. In this hardware the ON and OFF status of a line is denoted by LOGIC 1 and LOGIC 0 respectively. The status of all the lines is denoted through on-board LEDs which is interfaced with the FPGA kit. The I/O pins of the FPGA chip are configured and port mapped accordingly. The Verilog program for 33 - bus single feeder system is implemented in Verilog through SPARTAN 3 FPGA (Field Programmable Gate Array) kit (Figure 8). It uses a XILINX XC3S400 chip for processing. The XC3S400 FPGA chip is embedded in a kit with peripheral ICs and components for research and development purpose.

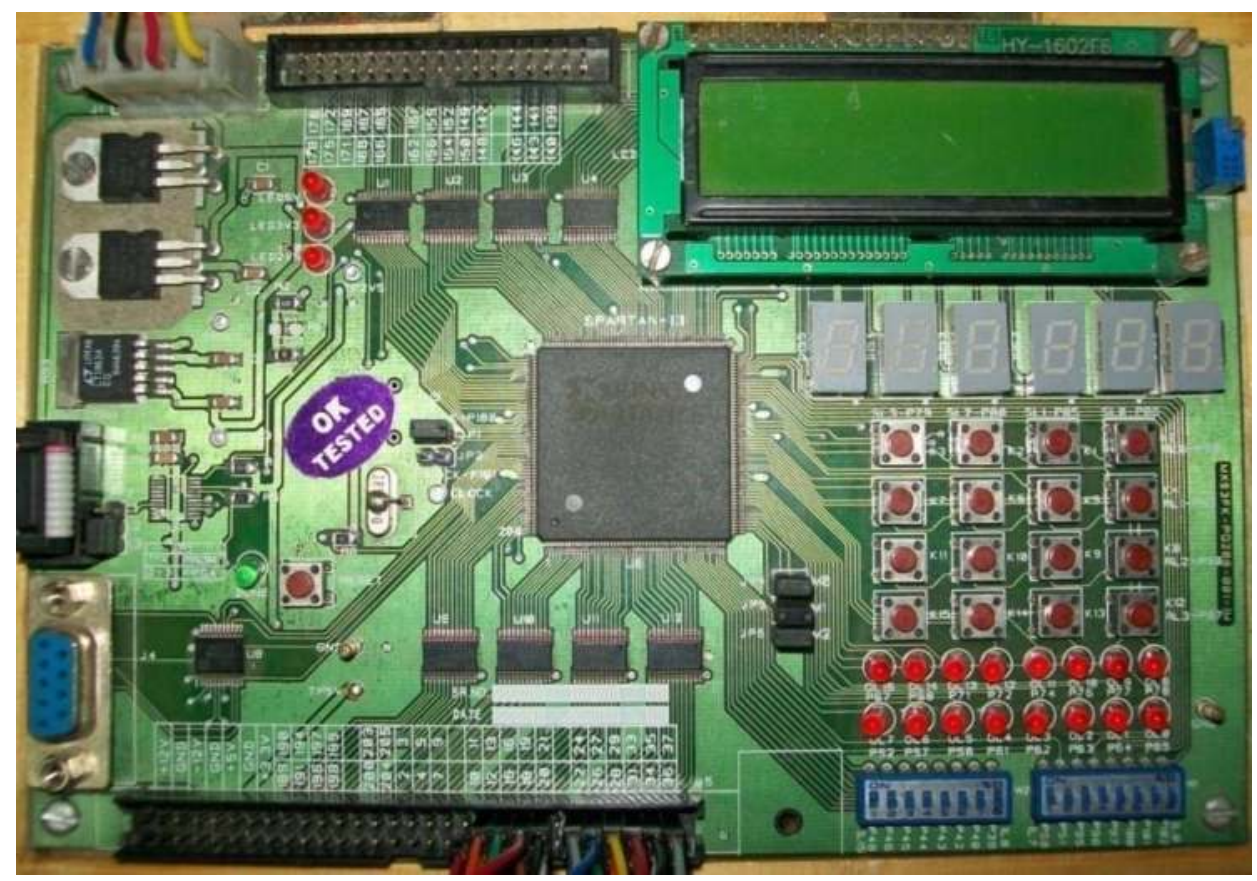

Fig. 8. XILINX SPARTAN3 FPGA Kit

A Verilog program is written using XILINX ISE software to program the FPGA and display the status of lines as output based on the outage line which is given as input. The chip is programmed using XILINX ISE navigator tool. The FPGA kit used in this project consists of sixteen input switches and sixteen output LEDs (Figure 9). In addition the kit also has a 34 pin FRC and 60 pin FRC connector for 94 I/Os. Using these additional pins, the status of other switches (S17 - S37) are indicated in a bread board (Figure 10). 


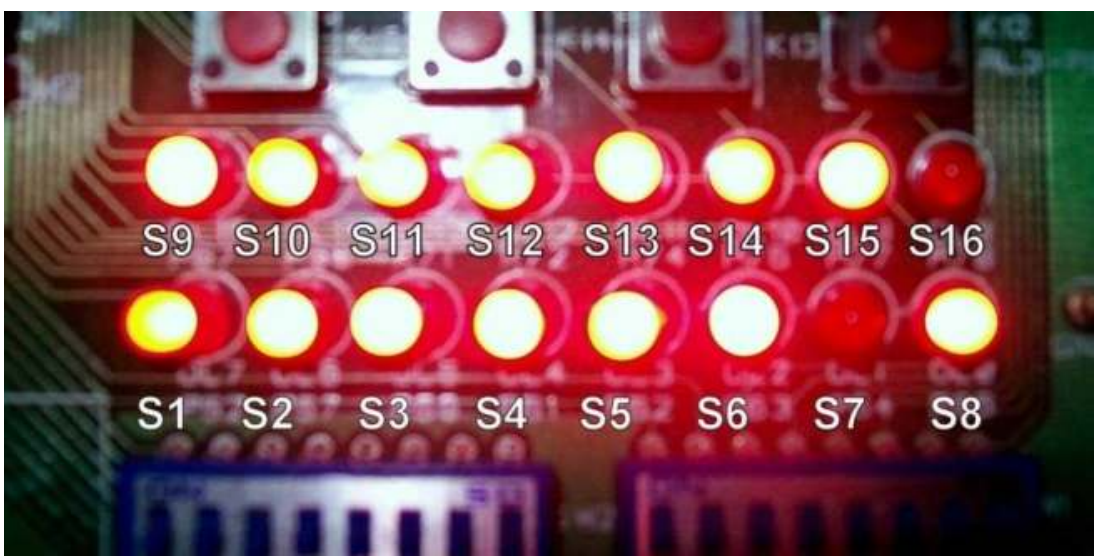

Fig. 9. Onboard LEDs displaying status of lines S1 to S16

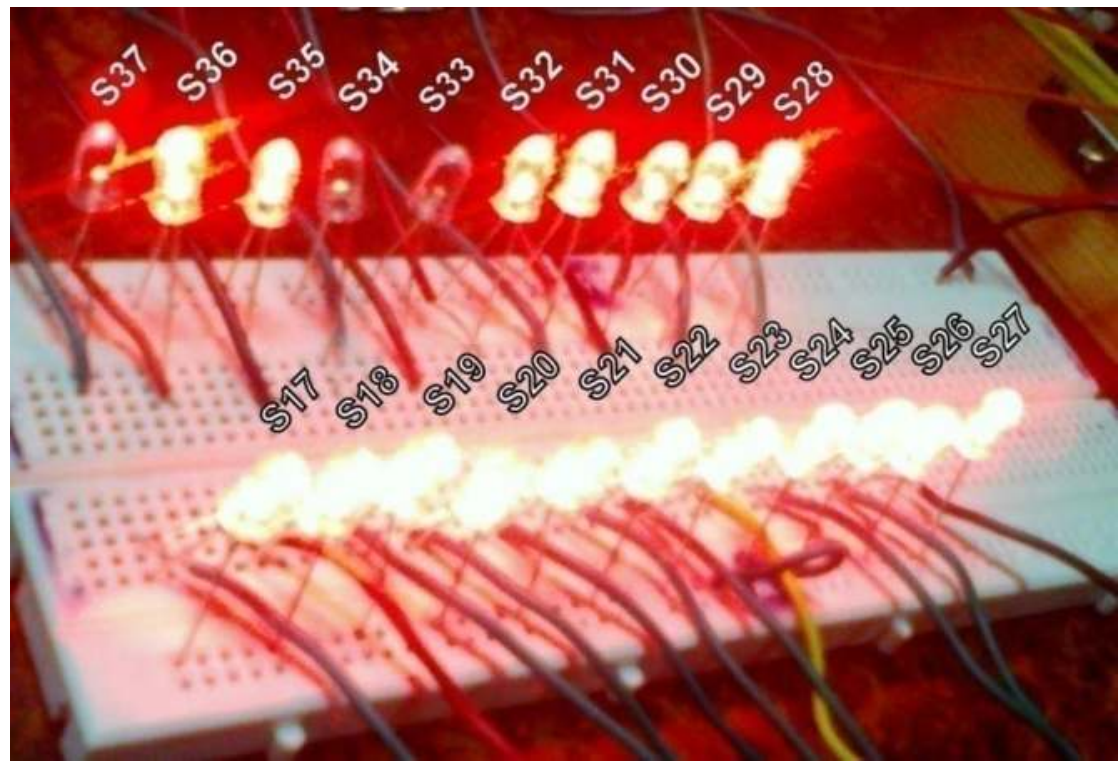

Fig. 10. Additional LEDs displaying status of lines S17 to S37

\section{Conclusion}

A feeder reconfiguration method using MST for service restoration of radial distribution system is presented. From the important observations of the present study it could be concluded that:

- $\quad$ The out-of-service area is reduced to the maximum by the developed MST methodology

- The power losses of distribution systems are reduced by proper feeder reconfiguration. 
- In addition to power-loss reduction, the voltage profile is improved by the proposed method.

- Based on the methodology the feeder loads and load flow are performed each time, so that the effect of unbalanced power distribution network is also considered.

- It can be applied to distribution network of any size.

Test results obtained indicate that, this method results in better restoration plan when compared to other reference papers.

\section{References}

Adibi, M. M. and Kafka, R. J. (1991). Power System Restoration Issues, IEEE Computer Applications in Power, Vol. 4, No. 2, pp. 19-24.

Ali Shatnawi, M O Ahmad and M N Swamy, (1999) Scheduling of DSP data flow graphs onto multiprocessor for maximum throughput, IEEE 1999, pp 386 - 389.

Cavellucci and Lyra, (1997), Minimization of energy losses in electric power distribution system by intelligent search strategies, International transaction in operational research, vol. 4, no. 1 , pp $23-33$.

Fountas, N. A. Hatziargyriou, N. D. and Valavanisl, K. P. (1997). Hierarchical Time Extended Petri Nets As A Generic Tool For Power System restoration, IEEE Transactions on Power Systems, Vol. 12, No. 2, pp. 837-843.

Gregory Levitin, Shmuel Mazal-Tov and David Elmakis, (1995). Genetic algorithm for optimal sectionalizing in radial distribution systems with alternative supply, Electric Power Systems Research, Vol. 35, pp. 149-155.

Han - Ching kuo and Yuan Yih Hsu, (1993). Distribution System Load Estimation and Service Restoration using a Fuzzy Set Approach, IEEE Transactions on Power Delivery, Vol. 8, No. 4, pp. 1950-1957.

Hiroyuki Mork and Senji Tsuzuki, (1991), A fast method for topological observability analysis using minimum spanning tree technique, IEEE Transaction on power system vol. 6, no. 2, May 1991, pp $491-500$.

Hotta, K., Nomura, H., Takemoto, H., Suzuki, K., Nakamura, S. and Fukui, S. (1990). The Implementation of a Real-Time Expert System for a Restoration Guide in a Dispatching Center, IEEE Transactions on Power Systems, Vol. 5, No. 3, pp. 1032-1038.

Hoyong Kim, Yunseok ko and Kyung-Hee Jung, (1993), Artificial Neural-Network based Feeder Reconfiguration for Loss Reduction in Distribution Systems, IEEE Transactions on Power Systems, Vol. 8, No. 3, pp. 1356-1366.

Kaigui Xie, Jiaqi Zhou and R Billinton, (2003), Reliability evaluation algorithm for complex medium voltage electrical distribution networks based on the shortest path, IEE Proc.Gener. Transm. Distrib., vol. 150, no. 6, November 2003, pp 686 - 690

Lin Ming Jin and Shu Park Chan, (1989), An electrical method for finding suboptimal routes, ISCAS'89 IEEE pp 935 - 938.

Matsumoto, K., Sakaguchi, T., Kafka, R. J. and Adibi, M. M., (1992), Knowledge-Based Systems as Operational Aids in Power System Restoration, Proceedings of the IEEE, Vol. 80, No. 5, pp. 689-697. 
Michel Barbehenn, (1998), A note on the complexity of Dijkstra's algorithm for graphs with weighted vertices, IEEE Transactions on computers, vol. 41, no 2, feb 1998, pp 263.

Mohanty, I., Kalita, J., Das, S., Pahwa, A. and Buehler, E., (2003), Ant algorithms for the optimal restoration of distribution feeders during cold load pickup, Proceedings of the 2003 Swarm Intelligence Symposium, SIS '03, IEEE, pp. 132-137.

Morelato, A. L. and Monticelli, A. (1989) Heuristic Search Approach to Distribution System Restoration, IEEE Transactions on Power Delivery, Vol. 4, No. 4, pp. 2235-2241.

Nagata, T. and Sasaki, H., (2002), A Multi-Agent Approach to Power System Restoration, IEEE transactions on power systems, Vol. 17, No. 2, pp. 457- 462.

Partricia Amancio Vargas, Christiano Lyra Filho and Fernanado J Von Zuben, (2002), On line approach for loss reduction in electric power distribution networks using learner classifier systems, Springer, pp $181-196$.

Rahman, S., (1993), Artificial intelligence in electric power systems - a survey of the Japanese industry, IEEE Transactions on Power System, Vol. 8, No. 3, pp. 1211-1218.

Shun Lin Su, Charles H Barry and Chi Yuan Lo, (1994) A space efficient short finding algorithms, IEEE Transactions on computer aided design of integrated circuit \& systems, vol. 13, no. 8, August 1994, pp 1065 - 1068.

Si - Qing Sheng, Yun Cao and Yu Yao, (2009), Distribution Network Reconfiguration Based on Particle Swarm Optimization and Chaos Searching", Asia-Pacific Power and Energy Engineering Conference, APPEEC 2009, IEEE, pp. 1-4

Sudhakar T D, et. Al, (2010), "Prim's Algorithm for Loss Minimization and Service Restoration in Distribution Networks", International Journal of Electrical and Computer Engineering (IJEC), Volume 2, Number 1 (2010), pp. 43 - 62

Sudhakar T D, et. Al, (2010), “A Graph Theory - Based Distribution Feeder Reconfiguration for Service Restoration", International Journal of Power and Energy Systems, Vol. 30, No. 3, 2010, pp $161-168$

Sudhakar T D, et. Al, (2011), "Power System Reconfiguration based on Kruskal's Algorithm" IEEE conference ICEES 2011 Volume 1 Page No 234 - 240

Sudhakar T D, et. Al, (2011), "Power System Restoration using Reverse Delete Algorithm Implemented in FPGA", Second IET International Conference on Sustainable Energy and Intelligent System, Page : 373 - 378

Sudhakar T D, et. Al, (2011), "Restoration of Power Distribution Network - A Bibliographical Survey", European Transactions on Electrical Power, Vol. 21, pp 635 - 655

TianTian CAi and Qian Ai, (2005), Research of PMU optimal placement in power systems", International conference on system theory and scientific computation, pp 38 - 43, ISBN 960-845735-1

Toune, S., Fudo, H., Genji, T., Fukuyama, Y. and Nakanishi, Y., (1998), A reactive tabu search for service restoration in electric power distribution systems" The 1998 IEEE International Conference on Evolutionary Computation Proceedings, IEEE World Congress on Computational Intelligence, pp. 763-768.

Ying - Tung Hsiao and Ching - Yang Chien, (2000), Enhancement of Restoration Service in Distribution Systems Using a Combination Fuzzy - GA Method, IEEE Transactions On Power Systems, Vol. 15, No. 4, pp. 1394-1400. 
Yixin Yu, and Jianzhong Wu, (2002), Loads Combination Method Based Core Schema Genetic Shortest-path Algorithm For Distribution Network Reconfiguration, 2002 IEEE, pp 1729 - 1733. 


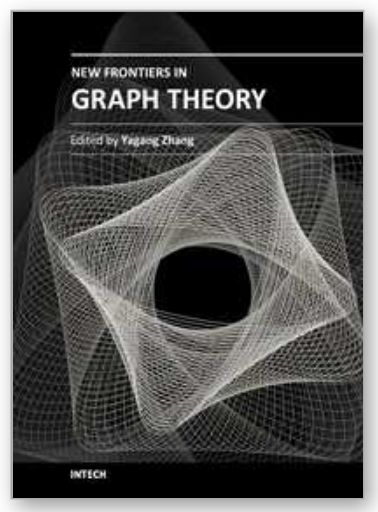

\author{
New Frontiers in Graph Theory \\ Edited by Dr. Yagang Zhang
}

ISBN 978-953-51-0115-4

Hard cover, 526 pages

Publisher InTech

Published online 02, March, 2012

Published in print edition March, 2012

Nowadays, graph theory is an important analysis tool in mathematics and computer science. Because of the inherent simplicity of graph theory, it can be used to model many different physical and abstract systems such as transportation and communication networks, models for business administration, political science, and psychology and so on. The purpose of this book is not only to present the latest state and development tendencies of graph theory, but to bring the reader far enough along the way to enable him to embark on the research problems of his own. Taking into account the large amount of knowledge about graph theory and practice presented in the book, it has two major parts: theoretical researches and applications. The book is also intended for both graduate and postgraduate students in fields such as mathematics, computer science, system sciences, biology, engineering, cybernetics, and social sciences, and as a reference for software professionals and practitioners.

\title{
How to reference
}

In order to correctly reference this scholarly work, feel free to copy and paste the following:

T. D. Sudhakar (2012). Power Restoration in Distribution Network Using MST Algorithms, New Frontiers in Graph Theory, Dr. Yagang Zhang (Ed.), ISBN: 978-953-51-0115-4, InTech, Available from: http://www.intechopen.com/books/new-frontiers-in-graph-theory/mst-algorithms-based-restoration-of-power-indistribution-network-

\section{INTECH}

open science | open minds

\section{InTech Europe}

University Campus STeP Ri

Slavka Krautzeka 83/A

51000 Rijeka, Croatia

Phone: +385 (51) 770447

Fax: +385 (51) 686166

www.intechopen.com

\section{InTech China}

Unit 405, Office Block, Hotel Equatorial Shanghai

No.65, Yan An Road (West), Shanghai, 200040, China

中国上海市延安西路65号上海国际贵都大饭店办公楼 405 单元

Phone: +86-21-62489820

Fax: $+86-21-62489821$ 
(C) 2012 The Author(s). Licensee IntechOpen. This is an open access article distributed under the terms of the Creative Commons Attribution 3.0 License, which permits unrestricted use, distribution, and reproduction in any medium, provided the original work is properly cited. 